\title{
Behavioral and Psychological Symptoms of Dementia in Alzheimer's Dementia: The Neuroimaging Correlates
}

\author{
Isha Ahluwalia ${ }^{1}$, G Prasad Rao $^{2}$, Chytanya Deepak P ${ }^{3}$, Monisha Reddy ${ }^{4}$
}

\begin{abstract}
Background: Behavioral and psychological symptoms of dementia (BPSD) is defined as"symptoms of disturbed perception, thought content, mood or behavior that frequently occur in patients with dementia." Behavioral and psychological symptoms of dementia is associated with significant caregiver burden, early institutionalization, and rapid cognitive decline. The worldwide prevalence of dementia is 5-7\%; whereas in India, it is reported to be $0.8-4 \%$. Alzheimer's dementia amounts to $60 \%$ of the total prevalence of dementia. We aim to study the neuroimaging correlates of BPSD in Alzheimer's dementia. Objective: To study the correlation between BPSD with white matter changes on neuroimaging in Alzheimer's dementia.

Materials and methods: It is a cross-sectional study with a sample size of 30 patients. The magnetic resonance imaging (MRI) changes were seen using volumetric analysis and white matter hyperintensity change using Fazekas scale, global cortical atrophy (GCA) score, medial temporal atrophy (MTA) score, and KOEDAM scale.

Results: It was found that clinical dementia rating (CDR) scale has positive correlation with Fazekas, GCA, and KOEDAM. Addenbrooke's cognitive examination (ACE-3) has correlation with GCA. On neuropsychiatric inventory (NPI) questionnaire, depression followed by nighttime behavior were the most common BPSD symptoms. Sociodemographic factors such as age $>65$ years, middle socioeconomic status (SES) population, and urban population have higher mean neuropsychiatric inventory-frequency and severity (NPI-F*S) scores.

Conclusion: Our study here in concordance with other studies worldwide gives a clue about the role of neuroimaging biomarkers in understanding BPSD in terms of its neuroimaging correlates and builds the scope of future studies in this domain. The study sheds light on the common presentation of BPSD, which adds to our knowledge in clinical assessment of Alzheimer's disease (AD) patients. Finally, it also adds to our understanding of the role of sociodemographic factors in predicting the at risk population.

Keywords: Alzheimer's dementia, Behavioral and psychological symptoms of dementia, Neuroimaging.

Indian Journal of Private Psychiatry (2019): 10.5005/jp-journals-10067-0042
\end{abstract}

\section{INTRODUCTION}

According to the International Classification of Diseases, Tenth Revision (ICD-10), dementia is a syndrome caused by the disease of the brain. It is usually of a chronic or progressive nature, with disturbance in multiple higher cortical functions, including memory, thinking, orientation, comprehension, calculation, learning capacity, language, and judgment. The worldwide prevalence of dementia is $5-7 \%$ which contributes to $4.1 \%$ of all disabilities adjusted life years. ${ }^{1}$ But in India it has been reported to vary from 0.8 to $4 \%$. Of this, AD accounts for $60 \%$, whereas vascular dementia accounts for $\sim 30 \%$ of the prevalence. ${ }^{2}$ It is estimated that the number of people living with dementia will almost double every 20 years, i.e., to 42.3 million in 2020 and 81.1 million in 2040. According to the International Psychogeriatric Association, behavioral and psychological symptoms of dementia (BPSD) is defined as "symptoms of disturbed perception, thought content, mood or behavior that frequently occur in patients with dementia". The pathogenesis of BPSD has not been clearly delineated, but it is probably the result of a complex interplay of psychological, social, and biological factors. There is still debt in our understanding of BPSD and its possible neuroimaging biomarkers; therefore, we have conducted a study to further understand BPSD with the help of neuroimaging correlates.

\section{A IM \\ Primary Objective}

To study the correlation between BPSD with white matter changes on neuroimaging in Alzheimer's dementia.
1,3,4 Department of Psychiatry, Asha Hospital, Hyderabad, Telangana, India ${ }^{2}$ Department of Psychiatry, Schizophrenia and Psychopharmacology Division, Asha Hospital, Hyderabad, Telangana, India

Corresponding Author: Isha Ahluwalia, Department of Psychiatry, Asha Hospital, Hyderabad, Telangana, India, e-mail: isha1804@gmail.com

How to cite this article: Ahluwalia I, Rao GP, Chytanya Deepak P, et al. Behavioral and Psychological Symptoms of Dementia in Alzheimer's Dementia: The Neuroimaging Correlates. Ind J Priv Psychiatry 2019;13(2):48-51.

Source of support: Nil

Conflict of interest: None

\section{Secondary Objective}

To study the sociodemographic factors and BPSD in Alzheimer's dementia.

\section{Materials and Methods}

This is a cross-sectional study conducted in the outpatient facility of tertiary care private hospital at Hyderabad, India. Thirty subjects who fulfilled the inclusion criteria were enrolled for the study. Informed consent was obtained from each participant, and if some participant lacked capacity for consent, consent was obtained from the nominated representative/first-degree relative of the patient.

\section{Inclusion Criteria}

- Age of $\geq 50$ years.

- According to ICD-10 diagnosed cases of AD with BPSD.

(0) The Author(s). 2019 Open Access This article is distributed under the terms of the Creative Commons Attribution 4.0 International License (https://creativecommons. org/licenses/by-nc/4.0/), which permits unrestricted use, distribution, and non-commercial reproduction in any medium, provided you give appropriate credit to the original author(s) and the source, provide a link to the Creative Commons license, and indicate if changes were made. The Creative Commons Public Domain Dedication waiver (http://creativecommons.org/publicdomain/zero/1.0/) applies to the data made available in this article, unless otherwise stated. 
- They will be right-handed as assessed by the modified Edinburgh handedness inventory. ${ }^{3}$

All subjects underwent routine investigations, neurocognitive assessment, and magnetic resonance imaging (MRI) brain dementia protocol and the required study parameters were obtained. The data were collected after approval from the ethics review committee board. The participants were compared on sociodemographic characteristics, performance on various neuropsychological tests, and neuroimaging scores. Statistically analysis was performed using the Statistical Package for the Social Sciences software version 16.0 (IBM Corp., Armonk, New York, USA). The data were analyzed using nonparametric correlation analysis. The data were screened for outliers. The correlation coefficient ranged from -1 to 1 and was tested for significance. If the $p$ value is less than 0.05 , correlation was considered as significant and two variables were said to be linearly correlated.

Tools used in the study:

- Sociodemographic data and clinical data sheet.

- Magnetic resonance imaging brain changes using volumetric analysis and white matter hyperintensity change using the Fazekas scale, global cortical atrophy (GCA) score, medial temporal atrophy (MTA) score, and KOEDAM scale.

- Psychiatric illnesses ruled out using MINI.

- The neuropsychiatric inventory (NPI) questionnaire ${ }^{4}$ was used to measure BPSD.

- Severity of dementia was understood using the clinical dementia rating (CDR) scale.

- Addenbrooke's cognitive examination.

- Disability assessment for dementia.

\section{Results}

We found that among the total number of patients $(n=30), 18$ patients were male with a mean NPI of 21.5 and 122 were female with an average NPI of 21 (Table 1).

Thirteen patients were less than 65 years of age and the rest (17) were more than 65 years of age (Table 2).

In all, 7 patients belonged to the upper class, 22 to middle class, and 1 to lower class, according to Kuppuswamy and Prasad socioeconomic scale (Table 3). Twenty-three patients were residents of urban areas and the rest living in rural areas (Table 4).

As shown in Table 5, only GCA score showed significant $p$ value when compared with ACE-3. However, when compared with CDR scale, Fazekas, GCA, and KOEDAM score of posterior atrophy, all had significant $p$ values, with the exception of the MTA score.

The percentage of various symptoms on NPI is mentioned in Table 6, with the highest number of patients having depression and only one patient showing elation. Hallucination was seen in $26 \%$ patients and only two patients showed disinhibition. The second most common symptom exhibited by the patients was irritability, with 20 patients showing the symptom.

The MRI of a 55-year male (Fig. 1) showed progressive cerebral atrophy over a period of 3-4 years. Patient had presented with BPSD which initially presented as depressive features progressing to apathy and unawareness during the course of follow-up of Alzheimer's dementia.

\section{Discussion}

Behavioral and psychological symptoms of dementia are commonly seen in neurocognitive illness. It is the most studied and found
Table 1: Sex ratio with mean neuropsychiatric inventory

\begin{tabular}{lll}
\hline Gender & Number of subjects & NPI-F*S (mean) \\
\hline Male & 18 & 21.5 \\
Female & 12 & 21 \\
Total & 30 & 21.2 \\
\hline
\end{tabular}

Table 2: Age ratio with mean neuropsychiatric inventory

\begin{tabular}{lll}
\hline Age (in years) & Number of subjects & NPI-F*S (mean) \\
\hline$<65$ & 13 & 17.92 \\
$>65$ & 17 & 23.7 \\
\hline
\end{tabular}

Table 3: Socioeconomic correlation

\begin{tabular}{lcc}
\hline Socioeconomic status & Number of subjects & NPI-F*S (mean) \\
\hline Upper & 7 & 13.14 \\
Middle & 22 & 24.46 \\
Lower & 1 & 1 \\
\hline
\end{tabular}

Table 4: Residential demographics

\begin{tabular}{lll}
\hline Residence & Number of subjects & NPI-F*S (mean) \\
\hline Urban & 23 & 23.21 \\
Rural & 7 & 14.57 \\
\hline
\end{tabular}

Table 5: Bivariate correlation between neurocognitive tests and neuroimaging scores

\begin{tabular}{lllll}
\hline & \multicolumn{4}{l}{ Neuroimaging scores } \\
\cline { 2 - 5 } & \multicolumn{3}{l}{$p$ values } & \\
\cline { 2 - 5 } Neurocognitive tests & Fazekas & GCA & MTA & KOEDAM \\
\hline ACE-3 & 0.247 & 0.002 & 0.705 & 0.066 \\
CDR & 0.042 & 0.025 & 0.994 & 0.022 \\
DAD & 0.067 & 0.015 & 0.348 & 0.001 \\
NPI-F*S & 0.622 & 0.907 & 0.987 & 0.417 \\
NPI-D & 0.383 & 0.016 & 0.499 & 0.074 \\
\hline
\end{tabular}

$p$ value $<0.05$ is significant

Table 6: Neuropsychiatric inventory parameters

\begin{tabular}{lc}
\hline NPI parameters & Percentage \\
\hline Delusion & 30 \\
Hallucination & 26 \\
Agitation/aggression & 40 \\
Depression/dysphoria & 73 \\
Anxiety & 56 \\
Elation/euphoria & 3 \\
Apathy/indifference & 53 \\
Disinhibition & 6 \\
Irritability/liability & 60 \\
Motor disturbances & 33 \\
Nighttime behavior & 66 \\
Appetite/eating & 36
\end{tabular}

to be widely associated with Alzheimer's dementia, ${ }^{4}$ and its etiopathogenesis is still poorly understood. Various studies have been conducted to study the commonly occurring symptoms of BPSD, their prevalence, and severity at different stages of dementia. ${ }^{1,2}$ However, not many studies have been conducted to assess the specific volumetric changes in brain and its association with frequency, severity, and pattern of BPSD. 

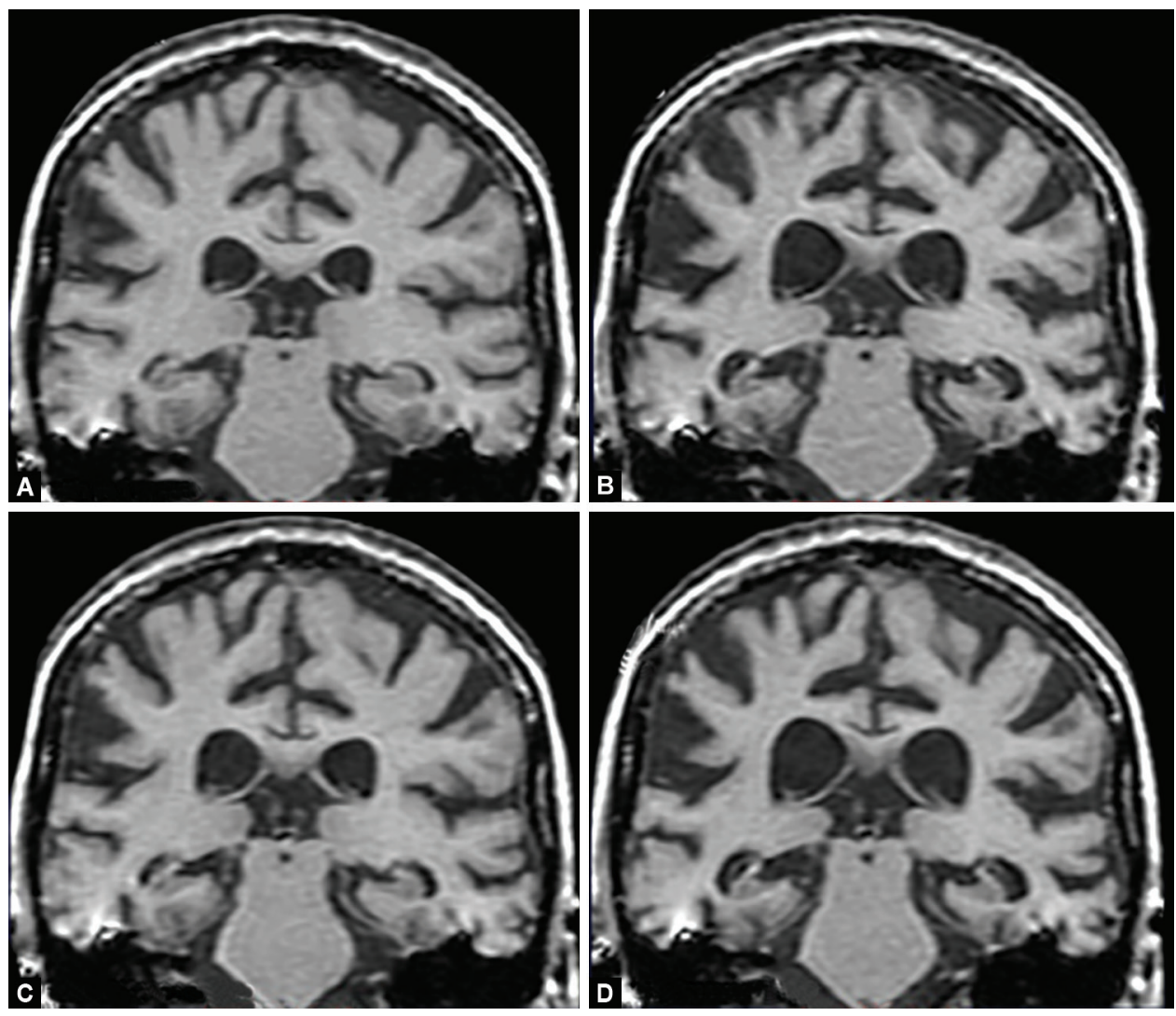

Figs $1 \mathrm{~A}$ to $\mathrm{D}$ : Magnetic resonance imaging showing progressive cerebral atrophy

In a study by Holthoff et al., ${ }^{5}$ based on MRI and fluorodeoxyglucose-positron emission tomography scan (FDG-PET), it was found that patients with apathy exhibited lower glucose metabolism in the left orbitofrontal cortex in comparison with other patients with no such symptoms [total study participants $n=53$; apathy $(n=17)$, not apathy $(n=17)$, depressed $(n=10)$, not depressed $(n=10)]$. Depressed group showed hypometabolism in the left prefrontal cortex and superior frontal cortex, when compared with subjects not depressed.

In another study, Bruen et al. ${ }^{6}$ studied subjects $(n=31)$ with Alzheimer's dementia, on MRI 1.5 T. Apathetic Alzheimer's disease (DA) subjects presented gray matter (GM) atrophy in the right inferior frontal gyrus, orbitofrontal cortex, bilateral dorsolateral prefrontal cortex, putamen, and head of the left caudate nucleus.

Serra et al., ${ }^{7}$ [total study participants $n=69 ; \mathrm{AD}(n=27)$ mild cognitive impairment (MCI) $(n=19)$ controls $(n=23)]$ in a group of patients with $\mathrm{MCl}$ and control subjects with $A D$, found decreased GM volume in the bilateral cingulate, which correlated with the severity of disinhibition and delusions in AD on 3 T MRI voxel based morphometry (VBM). Similarly, Berlow et al's ${ }^{8}$ study of AD patients $(n=37)$, the MRI findings showed that low volumes of white matter hyperintensity (WMH) were associated with disinhibition and high WMH volumes associated with anxiety.

Whitehead et al. ${ }^{9}$ found left medial orbitofrontal and superior temporal cortices were significantly thinner in those with paranoid delusions, i.e., $n=113$ patients, of which $n=90$ did not have delusions and $n=23$ had paranoid delusion. Female participants with paranoid delusions showed reduced cortical thickness in left medial orbitofrontal and left superior temporal regions.

In a large study by Palmqvist et al., ${ }^{10}$ it was demonstrated that of all the AD patients studied $[n=259 ; 8$ patients underwent MRI and all others computed tomography (CT)], the occurrence of lacunes in the left basal ganglia associated with increased risk of delusions and hallucinations and the presence of lacunes in the right basal ganglia associated with the risk of depressive symptoms. Increased global atrophy is associated with agitation.

In a study by Poulin et al., ${ }^{11}$ the level of motor aberrant behavior correlated with more severe amygdala atrophy in $A D$ subjects $(n=264)$ [mild AD sample $1(n=90)$ sample $2(n=174)]$, which is based on MRI 1.5 T.

In another large-scale study, Trzepacz et al..$^{2}$ based on the MRI findings showed atrophy in frontolimbic regions, right posterior cingulate, and left hippocampus were related to greater severity of agitation and aggression [i.e., total study patients $n=462$ which included $\mathrm{AD}(n=163), \mathrm{MCl}$ converters $(n=122)$ and $\mathrm{MCl}$ stable $(n=177)]$.

Based on structural and functional 3 T MRI, Balthazar et al. ${ }^{13}$ $(n=57)[\operatorname{AD}(n=37)$ and controls $(n=30)]$ suggested altered intrinsic connectivity of the anterior $\mathrm{SN}$ predicted behavioral symptoms in AD patients, e.g., hyperkinetic behavior. Hyperconnectivity of the salient network was observed in the anterior cingulate cortex and right insula.

In our study, it was found that nearly all patients had some behavioral or psychological symptoms on NPI, of which depression was the most common, followed by nighttime behaviors and the least being elation/euphoria and disinhibition, respectively; however, most other studies found apathy and delusions as the most common symptoms in the early progressive course of $A D \cdot{ }^{14}$ Also the corresponding MRI findings corroborated to the brain regions involved in the occurrence of these symptoms. ${ }^{15}$ 
The noteworthy finding here was correlation of neurocognitive assessment such as ACE-3 and CDR with neuroimaging scores such as Fazekas, GCA, MTA, and KOEDAM. It was particularly found that CDR scale correlated positively with Fazekas, GCA, and KOEDAM, which means as the performance on CDR deteriorates, the severity of neuroimaging scores increases. Similarly, ACE-3 had a positive correlation ( $p$ value of less than 0.05 ) with GCA score. These findings can give us the scope for future investigation in terms of the role of neuroimaging scores in predicting the development of BPSD symptoms and planning early targeted intervention in $A D$ and BPSD.

We also studied whether BPSD varies with sociodemographic profile, and it was found that those above 65 years of age had higher mean value of BPSD than those below 65 years of age. No significant difference was observed when compared with gender. It was found that middle SES participants had higher mean value of BPSD as opposed to the upper and lower SES subjects. Finally it was found that urban patients had more mean value of BPSD compared to the rural patients.

\section{Conclusion}

Our study here in concordance with other studies worldwide gives a clue about the role of neuroimaging biomarkers in understanding BPSD in terms of its neuroimaging correlates and builds the scope of future studies in this domain. ${ }^{15}$ The study sheds light on common presentation of BPSD which adds to our knowledge in clinical assessment of AD patients. Finally, it also adds to our understanding of the role of sociodemographic factors in predicting the at risk population.

The BPSD in Alzheimer's dementia still has a lacunae in our understanding of its definite etiopathogenesis; and thus, there is a barrier to predicting its course in disease and planning definite intervention. A worldwide more comprehensive prospective largescale studies of neurobiomarkers might be a breakthrough and in the future might help us in improving our understanding of the course of the disease progression.

Limitations of our study:

- The study was conducted on a small sample population.

- We could not study BPSD in other dementias such as frontotemporal dementia, vascular dementia, dementia of Lewy bodies, and Parkinson's dementia.

- Our study did not include other neurobiomarkers such as FDG-PET and single photon emission computed tomography (SPECT) scans and solely relied on MRI neuroimaging scores.

- We did not study BPSD in $\mathrm{MCl}$, which is a gray area in course of development of dementia.

\section{References}

1. Prince M, Bryce R, Albanese E, et al. The global prevalence of dementia: a systematic review and metaanalysis. Alzheimers Dement J Alzheimers Assoc 2013;9(1):63.e2-75.e2. DOI: 10.1016/j.jalz.2012.11.007.

2. Kalaria RN, Maestre GE, Arizaga R, et al. Alzheimer's disease and vascular dementia in developing countries: prevalence, management, and risk factors. Lancet Neurol 2008;7(9):812-826. DOI: 10.1016/S1474-4422(08)70169-8.

3. Raczkowski D, Kalat JW, Nebes R. Reliability and validity of some handedness questionnaire items. Neuropsychologia 1974;12(1):43-47. DOI: 10.1016/0028-3932(74)90025-6.

4. Pinto C, Seethalakshmi R. Behavioral and psychological symptoms of dementia in an Indian population: comparison between Alzheimer's disease and vascular dementia. Int Psychogeriatr 2006;18(1):87-93. DOI: $10.1017 /$ S104161020500311X.

5. Holthoff VA, Beuthien-Baumann B, Kalbe E, et al. Regional cerebral metabolism in early Alzheimer's disease with clinically significant apathy or depression. Biol Psychiatry 2005;57(4):412-421. DOI: 10.1016/j.biopsych.2004.11.035.

6. Bruen PD, McGeown WJ, Shanks MF, et al. Neuroanatomical correlates of neuropsychiatric symptoms in Alzheimer's disease. Brain 2008;131(Pt 9):2455-2463. DOI: 10.1093/brain/awn151.

7. Serra L, Perri R, Fadda L, et al. Relationship between cognitive impairment and behavioural disturbances in Alzheimer's disease patients. Behav Neurol. 2010;23(3):123-130. DOI: 10.1155/2010/528694.

8. Berlow YA, Wells WM, Ellison JM, et al. Neuropsychiatric correlates of white matter hyperintensities in Alzheimer's disease. Int J Geriatr Psychiatry 2010;25(8):780-788. DOI: 10.1002/gps.2418.

9. Whitehead D, Tunnard C, Hurt C, et al. Frontotemporal atrophy associated with paranoid delusions in women with Alzheimer's disease. Int Psychogeriatr 2012;24(1):99-107. DOI: 10.1017/S1041610211000974.

10. Palmqvist $S$, Sarwari A, Wattmo C, et al. Association between subcortical lesions and behavioral and psychological symptoms in patients with Alzheimer's disease. Dement Geriatr Cogn Disord 2011;32(6):417-423. DOI: 10.1159/000335778.

11. Poulin SP, Dautoff $R$, Morris JC, et al. Amygdala atrophy is prominent in early Alzheimer's disease and relates to symptom severity. Psychiatry Res 2011;194(1):7-13. DOI: 10.1016/j.pscychresns.2011.06.014.

12. Trzepacz PT, Yu P, Sun J, et al. Comparison of neuroimaging modalities for the prediction of conversion from mild cognitive impairment to Alzheimer's dementia. Neurobiol Aging 2014;35(1):143-151. DOI: 10.1016/j.neurobiolaging.2013.06.018.

13. Balthazar MLF, Pereira FRS, Lopes TM, et al. Neuropsychiatric symptoms in Alzheimer's disease are related to functional connectivity alterations in the salience network. Hum Brain Mapp 2014;35(4):1237-1246. DOI: 10.1002/hbm.22248.

14. Zhao Q-F, Tan $\mathrm{L}$, Wang $\mathrm{H}-\mathrm{F}$, et al. The prevalence of neuropsychiatric symptoms in Alzheimer's disease: systematic review and meta-analysis. J Affect Disord 2016;190:264-271. DOI: 10.1016/j.jad.2015.09.069.

15. Alves GS, Carvalho AF, de Amorim de Carvalho L, et al. Neuroimaging findings related to behavioral disturbances in Alzheimer's disease: a systematic review. Curr Alzheimer Res 2017;14(1):61-75. DOI: 10.2174/ 1567205013666160603010203. 